\title{
Posdesarrollo como herramienta para el análisis crítico del desarrollo
}

\section{Eduardo Gudynas*}

Resumen. A principios de la década de 1990 comenzó a emitirse un nuevo tipo de críticas al desarrollo que planteaban un cuestionamiento de fondo a esa categoría y a sus diversas manifestaciones. Desde esa óptica es posible considerar diferentes vertientes analíticas. La primera denuncia su fracaso generalizado y postula la necesidad de abandonarlo como discurso y política. La segunda afirma que representa una creencia, mito o religión occidental que se impone a otras culturas. La tercera lo cuestiona: toma como problema central el papel del crecimiento económico y demanda, en contraste, el decrecimiento. La cuarta, denominada posdesarrollo, lo valora como un discurso que expresa premisas como la modernización o la apropiación de la naturaleza, desde una pretensión de verdad universal, por lo que se subordinan otras culturas. Por tanto, la reconstrucción de los estudios críticos del desarrollo recurre a cuatro dimensiones analíticas: acciones específicas, programas de desarrollo sectorial, grandes familias y raíces en cuanto a las concepciones y sensibilidades.

Palabras clave: desarrollo, crítica, posdesarrollo, estudios críticos del desarrollo, critical root analysis.

* Secretario ejecutivo del Centro Latino Americano de Ecología Social, Uruguay. 


\section{Post-development as Tools \\ of a Critical Analysis of Development}

Abstract. At the beginning of the 1990s a new kind of criticism of development began to emerge that posed probing questions of the concept and its many expressions. From that perspective, we can consider differents analytical approaches. The first exposes its broad failure and proposes the need to abandon the concept as both discourse and policy. The second asserts that it represents a belief, myth or western religion that is imposed on other cultures. The third calls into question the central role of economic growth and demand, but not degrowth. The fourth, labelled «post-development», considers the rhetoric that values assumptions such as modernization or the conquest of nature, from a pretension of universal truth, to which other cultures are subordinate. The rebuilding of critical studies on development can draw upon, therefore, four analytical dimensions: specific actions, sectoral development programs, large families and roots pertaining to conceptions and sensitivities.

Keywords: development, criticism, post-development, critical development studies, critical root analysis. 


\section{Introducción}

A principios de la década de 1990 se consolidó un nuevo tipo de críticas al desarrollo que cuestionaba a esa categoría en sí misma y en todas sus manifestaciones. Bajo esa perspectiva se encuentran al menos cuatro énfasis: a) unos denunciaron el fracaso del desarrollo en cualquier expresión y la necesidad de abandonarlo (Esteva, 1992); b) otros afirmaron que era esencialmente una creencia, mito o religión occidental que se imponía a diferentes culturas (Rist, 1997); c) unos más lo cuestionaron enfocándose en el papel del crecimiento económico como un problema central, y desde allí postularon el decrecimiento (Latouche, 2009b); d) una corriente conocida como posdesarrollo, en la formulación de su promotor más conocido, Arturo Escobar, sostiene que debía ser considerado como un discurso que expresaba premisas como la modernización o la apropiación de la naturaleza, que era revestido como verdad universal, con lo cual se subordinaban otras culturas (Escobar, 1995).

Un examen más detallado del posdesarrollo muestra que por lo menos deben distinguirse dos etapas en las proposiciones de Escobar: en la primera se enfatiza al posdesarrollo como un análisis crítico; en la segunda, además de esa crítica, se le otorga más atención a distintas alternativas.

\section{El posdesarrollo como crítica}

El posdesarrollo inicial como crítica se inspiró en el posestructuralismo, especialmente en los análisis de los discursos promovidos por Michael 
Foucault; por lo tanto, un término más correcto debería ser «crítica posestructuralista» al desarrollo (una introducción a esta corriente se encuentra en Belsey, 2002; véase además Gibson-Graham, 2000). En tal contexto, los discursos del desarrollo no sólo incluyen los enunciados, sino también acciones concretas, instituciones que lo promueven y los modos de legitimación. De esa manera, deberían analizarse cuestiones como las ideas básicas en un plan de desarrollo, las agencias estatales por las cuales se lo implanta, las acciones que promueve y los modos de legitimación.

Ese tipo de crítica se orientaba a los aspectos básicos y comunes que se mantenían en distintas expresiones del desarrollo, y que permitían sostenerlo como una categoría universal que debería ser imitada en todos los países. La imposición de la idea de desarrollo a imagen de los países industrializados simultáneamente relegó a los demás a la condición de subdesarrollados. Por tanto, el desarrollo es simultáneamente tanto imposición de unos saberes como exclusión de otros.

El posdesarrollo permitió evidenciar una serie de conceptos básicos que sustentan la idea de desarrollo. Este sería un proceso universal, de tipo progresivo, en esencia positivo, que discurre linealmente. El principal motor sería el crecimiento económico, defendido como un principio a perpetuidad, y que a su vez genera el bienestar material de las personas, avances sociales, culturales y políticos. Así, el desarrollo defiende diferentes versiones de la modernización.

De una manera u otra el desarrollo entiende que sociedad y naturaleza están separadas. Se niegan o minimizan los impactos ambientales y sociales, y se defiende un optimismo científico-técnico. El énfasis económico del desarrollo genera una creciente mercantilización del entorno y de las relaciones sociales, ancladas en un estilo de vida y consumo occidental. Se 
refuerza el consumismo e incluso se imita una estética occidental. Asimismo, se mantienen posturas patriarcales de diverso tipo, lo que subordina e invisibiliza a las mujeres.

En su aplicación más estricta, un análisis desde el posdesarrollo considera las formas de saber (por ejemplo, la delimitación de disciplinas, las condiciones de validez), las subjetividades involucradas, los modos de representación de esos discursos (incluyendo las instancias donde se apela a la violencia o a las resistencias), y las dinámicas de poder que cruzan todas esas esferas, desde el papel de los expertos a las demandas o resistencias locales ante el desarrollo (Escobar, 1995, 2012). De esta manera, se determinan los criterios de verdad y falsedad sobre qué es el desarrollo, las razones para concebirlo como un proceso positivo, las concepciones aceptables sobre sus ideas constitutivas (tales como bienestar, eficiencia, crecimiento), e incluso las formas por las cuales interpretamos nuestras relaciones con el entorno social y natural.

Como se aprecia, la idea de desarrollo no está restringida a cuestiones económicas, sino que se derrama en las dimensiones sociales, culturales y políticas, e incluso en las sensibilidades y estéticas personales. Esta crítica posdesarrollista muestra que si bien el desarrollo no es un campo unificado ni tiene un significado preciso, se repiten atributos básicos y aparecen procesos de organización, legitimación y acción que son análogos. El desarrollo aparece entonces como un tipo de relacionalidad, que agrupa algunas ideas y prácticas pero excluye otras. A su sombra han surgido conceptos de enorme influencia, como capital humano o capital natural, o se han redefinido otros, como eficiencia o inequidad, todos los cuales se pueden pensar de unas pocas maneras. 


\section{El posdesarrollo como crítica y espacio de alternativas}

En su etapa inicial el posdesarrollo permitió hacer una distinción clave: por un lado, existirían los «desarrollos alternativos»y, por otro lado, las «alternativas al desarrollo» (véase Escobar, 1995:215). Los primeros son debates sobre ajustes instrumentales o diversas formas de organizar el desarrollo; no están en discusión sus cimientos conceptuales. Corresponden, por ejemplo, discusiones acerca de los mejores modos de alimentar el crecimiento económico, el papel del mercado o del Estado.

Las segundas, como alternativas a cualquiera de las visiones del desarrollo, quedaron en evidencia gracias a las críticas del posdesarrollo. En sus formulaciones originales, el posdesarrollo entendía que esas alternativas se orientaban a conformar un discurso de la diferencia o a rescatar los ensayos y resistencias que partían desde los movimientos del Sur; pero a partir de mediados de la década de 2000, poco a poco, se propagó la confusión.

Varios interpretaron que el prefijo «pos» aludía a un futuro desarrollo que superaría las limitaciones de los actuales o incluso abarcaría las posiciones antidesarrollo (como las de Gustavo Esteva). Así se debilitaba el vínculo directo con el posestructuralismo y se mezclaban algunos desarrollos alternativos con alternativas al desarrollo. Escobar contribuyó a esta confusión al sumar al posdesarrollo la tarea de crear nuevos discursos y representaciones, diversificar los agentes de producción del conocimiento o apoyar resistencias (Escobar, 2005). Recientemente, le sumó cuestiones como los «discursos de transición» (Escobar, 2012). Sin duda en su obra inicial estaba presente cierta superposición entre el cuestionamiento de inspiración posestructuralista y la imaginación de alternativas, pero todo ello se agudizó en esa segunda etapa (véase Ziai, 2007). 
Tal ampliación, todavía en marcha, generó una mayor adhesión al rótulo posdesarrollo, sobre todo desde militantes sociales, pero a costa de perder especificidad analítica. A su vez, si bien una crítica posdesarrollista es potente, no es suficiente para generar alternativas, éstas precisan de instrumentos y reflexiones propios (Gudynas, 2014b).

\section{Reacciones y constancias frente al posdesarrollo}

El posdesarrollo en su primera etapa fue objeto de muchos cuestionamientos. Entre las objeciones se señalaba la incapacidad de entender la heterogeneidad en las prácticas del desarrollo o la romantización de los movimientos sociales, y por eso no faltaron quienes consideraran que sólo era una retórica antimoderna (véase Nederveen, 2000). A juicio de varios analistas, el problema no era el desarrollo en sí mismo, sino sus aplicaciones capitalistas o la persistencia de la pobreza. También se señalaron problemas metodológicos, entre ellos que los ejercicios iniciales en realidad eran una expresión parcial o empobrecida del posestructuralismo (Ziai, 2004).

Sin embargo, el posdesarrollo se mantuvo como un espacio de análisis crítico y contestación al desarrollo, e incluyó algunos nuevos aportes que seguían la perspectiva posestructuralista de forma más rigurosa. Simultáneamente, desde el campo de los estudios del desarrollo las reacciones de rechazo iniciales dieron paso a reflexiones más rigurosas que aceptaban algunas de las advertencias del posdesarrollo (un resumen de esta situación en Ziai, 2015).

Es pertinente indicar ciertas limitaciones y precisiones: la crítica del posdesarrollo, más allá de invocar vínculos directos con determinados movimientos sociales, en realidad discurrió como un ejercicio académico 
con débiles conexiones con las grandes transformaciones políticas. Esto fue evidente en América del Sur, desde donde surgió uno de los cuestionamientos al desarrollo más potentes, conocido como Buen Vivir. Tal concepción es tanto una crítica radical al desarrollo como la apertura a alternativas poscapitalistas y postsocialistas. Es una postura que surgió por fuera de ejercicios académicos, y como resultado de prácticas sociales y políticas ciertamente heterodoxas y desde una notable diversidad de actores (sobresalen militantes indígenas) (Gudynas, 2014b). Esas críticas muestran semejanzas importantes con el posdesarrollo inicial.

Paralelamente, la severa crisis económico-financiera de 2007-2008, que para algunos anunciaba el fin del capitalismo, en realidad no mermó la prevalencia de las ideas del desarrollo, sólo han cambiado sus componentes y expresiones. Ejemplos de ello son la enorme diversidad de debates alrededor de los Objetivos de Desarrollo del Milenio y los más recientes Objetivos de Desarrollo Sostenible. El posdesarrollo desempeñó un papel limitado en dichas discusiones, pero esa permanencia coincide con su señalamiento de que el desarrollo está arraigado a determinados componentes que se reproducen una y otra vez.

De la misma manera, un análisis de las estrategias de desarrollo más recientes desde inicios del siglo XXI manifiesta una notable variedad en sus expresiones instrumentales, pero una gran constancia en los componentes básicos. Regímenes muy distintos, como pueden ser las administraciones europeas que defienden ajustes neoliberales o los planes de desarrollo que en China formula el Partido Comunista, sin duda son diferentes, pero a la vez evidencian elementos básicos comunes.

Un examen más detallado para el caso de América del Sur es todavía más impactante, ya que allí se han ensayado en un corto periodo múltiples 
estrategias de desarrollo: desde posturas conservadoras (por ejemplo en Chile, Colombia y Perú), a ensayos heterodoxos que invocaban un nuevo socialismo del siglo XXI (en Bolivia, Ecuador y Venezuela), pasando por economías de mercado bajo cierta regulación estatal (en Brasil y Uruguay). Las legitimaciones de estas variedades de desarrollo en unos casos aluden a concepciones neoclásicas ortodoxas, pero en otras se cita a Marx o Lenin (como hacen el presidente de Ecuador, Rafael Correa, o el vicepresidente de Bolivia, Álvaro García Linera).

Esas experiencias, que son objeto de intensos debates son diversas; si bien hay éxitos en algunas áreas, es impactante observar que se repiten elementos básicos como el apego al crecimiento económico o la ambición de la modernización. Por lo tanto, dichas bases conceptuales son previas a las distintas corrientes ideológico políticas en cualquiera de esos países. Estamos lidiando con concepciones y sensibilidades comunes a ideologías políticas y filosóficas propias de la modernidad. Esto es justamente otro de los puntos que señala el posdesarrollo en su versión original.

\section{Repensando los estudios críticos del desarrollo}

La diversidad de expresiones instrumentales del desarrollo aun en contextos de crisis y cambios políticos, deja evidencia de la permanencia de concepciones y sensibilidades básicas, por lo que es imprescindible hacer abordaje crítico en la misma línea del posdesarrollo. Recordemos que bajo el calificativo «crítica» se alude a los cuestionamientos sobre las diferentes manifestaciones del desarrollo. Para algunos, esa crítica parte de compromisos normativos tales como analizar ciertos sujetos (los efectos 
del desarrollo sobre los más pobres o la situación del Tercer Mundo) o temas (justicia social, equidad, etcétera). Para otros, la crítica al desarrollo es un medio para cuestionar escuelas de pensamiento económico o político (unos cuestionan al capitalismo y otros al socialismo).

En general, en los últimos años se ha vuelto común entender como estudios críticos del desarrollo a los que parten desde una mirada de «izquierda», incluyendo aportes del marxismo, la poscolonialidad, la ecología, el feminismo y otros (al respecto véase Veltmeyer et al., 2011; Schuurman, 2009; Kothari, 2005). Numerosos abordajes son herederos de la teoría crítica de la Escuela de Frankfurt, al rechazar el positivismo y defender los saberes social e históricamente enmarcados, que se orientan en prácticas de transformación.

Un problema, tal vez más grave, es que conocidas posturas críticas se enfocan en el capitalismo y no en el desarrollo. En muchos casos esas corrientes desnudan la cara oscura y las contradicciones del capitalismo, pero como no logran abordar las raíces del desarrollo, no presentan cuestionamientos a ese nivel ni alternativas para superarlo (un ejemplo de ello es Harvey, 2015). Todavía más problemático es el intento de recolocar al Buen Vivir como una nueva variedad de desarrollo socialista, tal como promueven actores vinculados a los gobiernos de Ecuador y Bolivia, para despojarlo de su crítica radical.

La cuestión clave es que gran parte de estos abordajes terminan siendo discusiones entre distintos tipos o «familias» de desarrollo, donde se atacan unas versiones mientras se postulan otras alternativas mejores. Por lo tanto, ese tipo de estudios se autolimita al campo del desarrollo. Munck sostiene que «no es posible superar el reto de la teoría crítica del desarrollo alejándose o abandonando el desarrollo. En cambio, el resto es imaginar y 
operar el desarrollo de una forma diferente» (Munck, 2011:76-77). Esa perspectiva, que la crítica y la alternativa tienen que estar dentro del desarrollo, es la que precisamente deja en evidencia el posdesarrollo.

Sin duda, los análisis críticos del desarrollo desde una sensibilidad de izquierda son útiles para lidiar con ciertas familias de variedades de desarrollo, pero son insuficientes. Para completar el campo de la crítica, haciéndola rigurosa y abarcadora, es necesario ir hacia niveles más profundos.

\section{Niveles en los estudios críticos sobre los desarrollos}

Es posible identificar al menos cuatro niveles en los que operan las evaluaciones críticas sobre los desarrollos (identificados como 3, 2, 1 y 0). El nivel tres es el más común y superficial; corresponde a los análisis sobre acciones de desarrollo específicas, como puede ser un programa de crédito rural o un plan de construcción de viviendas populares. Respecto al nivel dos, compete a los programas de desarrollo sectoriales; las acciones indicadas en el ejemplo anterior atañen a su vez a entendimientos sobre qué es el desarrollo rural o el desarrollo humano. Es obvio que en ese nivel, desde los programas sectoriales se derivan distintos planes y acciones hacia el tercer estrato. En estos dos niveles operan los análisis de desarrollo más conocidos, que sopesan la idoneidad de los instrumentos económicos utilizados, las diferencias entre los resultados esperados y los obtenidos, la generación de empleo, la promoción del crecimiento económico, etcétera.

El nivel uno concierne a una evaluación dentro de alguna de las grandes familias del desarrollo, como puede ser el desarrollo capitalista. En él 
se aplican instrumentos de los estudios críticos sobre el desarrollo comprometido con sectores populares o con temas como la justicia social, como se indicó arriba. Un análisis usual en este primer nivel es cuestionar al desarrollo como «ideología», donde la crítica se hace desde posturas filosófico-políticas (por ejemplo, los neoliberales atacando la pretensión de planificación estatal en el desarrollo o los socialistas reclamando el control del mercado). Esta cuestión se repite para los análisis abarcadores del desarrollo que lo consideran como una «ideología», «paradigma» $\mathrm{o}$ «cultura».

Finalmente, existe un nivel cero que corresponde a las bases conceptuales y sensibles sobre las que se sostienen las ideas y prácticas del desarrollo, las «raíces» comunes a cualquiera de sus variedades. Este es el estrato más profundo, donde se ubican las raíces conceptuales del desarrollo. Sin duda existen articulaciones entre los niveles, ya que unos están embebidos en los otros. No obstante, los instrumentos críticos que se aplican en un nivel no necesariamente son los mejores para otro. Los estudios críticos del nivel uno pueden ser útiles en los niveles dos y tres, pero no son los más adecuados para el cero.

El posdesarrollo en su sentido inicial, como crítica posestructuralista, es apropiado para una evaluación del nivel cero. No es la mejor herramienta para estudiar, por ejemplo, la efectividad de una estrategia de desarrollo en particular, en especial por sus conocidas limitaciones para entender la heterogeneidad del desarrollo. A su vez, ese tipo de análisis permite apelar a otros instrumentos que complementen la crítica sobre ese nivel. Se requieren varias herramientas complementarias debido a las complejidades y barreras en los prejuicios, entendimientos y sensibilidades profundamente arraigados. 


\section{Herramientas para un análisis crítico de las raíces del desarrollo}

Para el nivel cero de los análisis, que corresponde a lo que podría llamarse las «raíces» en las concepciones y sensibilidades del desarrollo, se puede describir una caja de herramientas de análisis crítico de esas raíces del desarrollo. En él se encuentran los siguientes instrumentos: posdesarrollo y otros análisis posestructuralistas sobre discursos del desarrollo, deconstrucción, performatividad, ciertas etnografías del desarrollo, varias metodologías en la economía ecológica, ética ambiental en su tratamiento de la asignación de valores, estudios de género, epistemología crítica y algunos de los ensayos sobre las llamadas «aperturas ontológicas». Esta composición es la que resulta de nuestro trabajo en el Centro Latino Americano de Ecología Social (CLAES), en los últimos años (más abajo se comentan atributos de esas herramientas).

Es un análisis crítico de las raíces en el sentido que apunta a la base de las ideas, prácticas y sensibilidades del desarrollo en todas sus expresiones. No se refiere a una mera enumeración de errores, ni necesariamente es un medio para elaborar alternativas al desarrollo, aunque la creencia o necesidad de pensar opciones distintas a las actuales sigue siendo uno de los motores de esas críticas.

Bajo tal clasificación, el posdesarrollo sería un instrumento entre otros posibles con estos instrumentos. Difícilmente se podría defender la existencia de una herramienta que fuese más efectiva y completa en desentrañar el nivel cero en todos sus aspectos. Además, cada instrumento tiene una especificidad, donde los componentes que escapan a su consideración pueden ser abordados por otros instrumentos complementarios. En esa complementación se logra un abordaje más completo. 
Es posible mencionar aspectos destacados de algunos instrumentos. La deconstrucción permite identificar las condiciones jerárquicas y binarias que imponen determinadas ideas y sensibilidades, y que a su vez excluyen a otras. Asimismo, la performatividad sirve para reconocer prácticas repetidas mediante las cuales el discurso produce efectos a los que da nombre (estos dos instrumentos aparecen en Gibson-Graham, 2000). El desarrollo está repleto de circunstancias donde la actuación genera actos que son defendidos como desarrollo. Las nuevas etnografías del desarrollo abordan prácticas concretas en emprendimientos y sitios específicos, desentrañan cómo las procesan los actores locales, las redes de relaciones que se crean, las resistencias o reinterpretaciones que emergen (véase Mosse, 2005).

La economía ecológica provee instrumentos clave para aproximarse a la imposibilidad del crecimiento económico perpetuo, los reales metabolismos de las articulaciones entre sociedad y naturaleza, y los efectos ecológicos y económicos de los impactos ambientales (véase Martínez y Roca, 2000). Además, ayuda a repensar los esquemas de valoración al cuestionar las conmensurabilidades perfectas.

Relativo a la ética ambiental, en especial la que aborda los valores propios entre los no humanos, sean especies vivas o ecosistemas, es fundamental para desentrañar la base valorativa de los desarrollos propios de la modernidad. En este campo se observan abordajes heterodoxos que incorporan sensibilidades de algunos pueblos indígenas (Gudynas, 2014a), como reflexiones más formales inspiradas, entre otros, en Naess (2016).

Muchos de los instrumentos de arriba implican otros modos de concebir la generación de conocimientos, y por ello se articulan con ciertas epistemologías críticas. A modo de ejemplo se puede citar a Donna Haraway (2004) por su postura antiesencialista y feminista, donde la identidad se 
extiende sobre otros que son considerados radicalmente distintos. Se rescatan también los aportes de la colonialidad del poder y del saber (inspirados en Quijano, 2000). Un producto de la modernidad son las bases del desarrollo, las cuales generan saberes y sensibilidades que se han construido en un entramado de poder, y que a la vez subalternan otros saberes y sensibilidades.

Las aperturas ontológicas permiten aproximarse a otras formas de entender y sentir lo que se considera como el mundo en que se vive (en el sentido de De la Cadena, 2014). Aquí se encuentran instrumentos relevantes para ir más allá de la gran división sociedad-naturaleza, son esenciales para los desarrollos convencionales porque rescatan otras cosmovisiones; destacan los aportes de Viveiros de Castro (2004) y Descola (2012) desde la antropología.

El surgimiento de ese conjunto de herramientas para el análisis de los fundamentos abre enormes potencialidades para una nueva generación de estudios críticos en el desarrollo. Aquellas se están aplicando en diversos sitios, y en varios casos son utilizadas directamente desde los movimientos sociales; todas tienen la potencialidad de promover alternativas que eviten volver a caer en los claroscuros del desarrollo.

\section{Referencias}

Belsey, Catherine (2002), Post-structuralism. A very short introduction, Oxford, Oxford University Press.

De la Cadena, Marisol (13 de junio de 2014), «The Politics of Modern Politics Meets Ethnographies of Excess Through Ontological Openings», Cultural 


\section{EDUARDO GUDYNAS}

Anthropology, en https://culanth.org/fieldsights/471-the-politics-of-modernpolitics-meets-ethnographies-of-excess-through-ontological-openings

Descola, Philippe (2012), Más allá de naturaleza y cultura, Buenos Aires, Amorrortu. Escobar, Arturo (2012), «Prefece to the 2012 edition», en Encountering development. The making and unmaking of the Third World, Princeton, Princeton University Press, pp. 6-43.

(2005), «El 〈postdesarrollo〉 como concepto y práctica social», en Daniel Mato y Sarah Babb (coords.), Politicas de economía, ambiente y sociedad en tiempos de globalización, Caracas, Universidad Central de Venezuela, pp. 17-31. (1995), Encountering development. The making and unmaking of the Third World, Princeton, Princeton University Press.

Esteva, Gustavo (1992), «Development», en Wolfgang Sachs (ed.), The development dictionary, London, Zed, pp. 6-25.

Gibson-Graham, J.K. (2000), «Postsructural interventions», en Eric Sheppard \& Trevor Barnes, A companion to economic geography, Oxford, Blackwell Publishing, pp. 95-110.

Gudynas, Eduardo (2014a), Derechos de la Naturaleza. Etica biocéntrica y políticas ambientales, Lima, CooperAcción/RedGE/Centro Latinoamericano de Estudios Superiores.

(2014b), «El postdesarrollo como crítica y el Buen Vivir como alternativa», en Gian Carlo Delgado Ramos (coord.), Buena Vida, Buen Vivir: imaginarios alternativos para el bien común de la humanidad, México: Universidad Nacional Autónoma de México-Centro de Investigaciones Interdisciplinarias en Ciencias y Humanidades, pp. 61-95.

Haraway, Donna (2004), The Haraway reader, New York, Routledge.

Harvey, David (2015), Seventeen contradictions and the end of capitalism, London, Oxford University Press. 
Kothari, Uma (2005), «A radical history of development studies: individuals, institutions and ideologies», en Uma Kothari (ed.), A radical history of development studies. Individuals, institutions and ideologies, London, Zed Books, pp. 1-13.

Latouche, Serge (2009), La apuesta por el decrecimiento, Barcelona, Icaria.

Martínez Alier, Joan y Jordi Roca Jusmet (2000), Economía ecológica y política ambiental, México, Fondo Cultura Económica.

Mosse, David (2005), Cultivating development. An ethnography of aid policy and practice, Londres, Pluto.

Munck, Ronaldo (2011), «Teoría crítica del desarrollo», en Henry Veltmeyer, Ivonne Farah \& Igor Ampuero (eds.), Herramientas para el cambio: manual para los estudios críticos del desarrollo, La Paz, Centro de Investigación para el Desarrollo Sustentable/Oxfam, pp. 73-77.

Naess, Arne (2016), Ecology of wisdom, Londres, Penguin.

Nederveen Pieterse, Jan (2000), «After post-development», Third World Quaterly, 21(2), pp. 175-191.

Quijano, Anibal (2000), «Coloniality of power, eurocentrism and Latin America», Nepantla: views from the South, 1(3), pp. 533-580.

Rist, Gilbert (1997), The history of development. From western origins to global faith, Londres, Zed.

Schuurman, Frans (2009), "Critical development theory: moving out of the twilight zone», Third World Quarterly, 30(5), pp. 831-848.

Veltmeyer, Henry (2011), «Vías hacia el cambio progresivo y el desarrollo alternativo», en Henry Veltmeyer, Ivonne Farah \& Igor Ampuero (eds.), Herramientas para el cambio: manual para los estudios críticos del desarrollo, La Paz, Centro de Investigación para el Desarrollo Sustentable/Oxfam, pp. 351-358. 


\section{EDUARDO GUDYNAS}

Veltmeyer, Henry, Ivonne Farah \& Igor Ampuero (2011), Herramientas para el cambio: manual para los estudios críticos del desarrollo, La Paz, Centro de Investigación para el Desarrollo Sustentable/Oxfam.

Viveiros de Castro, Eduardo (2004), «Perspectivismo y multiculturalismo en la América indígena», en Alexandre Surralles y Pedro García Hierro (eds.), Tierra adentro. Territorio indígena y percepción del entorno, Lima, Grupo Internacional de Trabajo sobre Asuntos Indígenas, pp. 37-80.

Ziai, Aram (2004), «The ambivalence of post-development: between reactionary populism and radical democracy», Third World Quaterly, 25(6), pp. 1045-1060.

(2007), Exploring post-development. Theory and practice, problems and perspectives, Routledge, London.

(2015), «Post-development: premature burials and haunting ghosts», Development Change, 46 (4), pp. 833-854. 